\title{
Self-Organization of Motor-Propelled Cytoskeletal Filaments at Topographically Defined Borders
}

\author{
Alf Månsson, ${ }^{1}$ Richard Bunk, ${ }^{2}$ Mark Sundberg, ${ }^{1}$ and Lars Montelius ${ }^{2}$ \\ ${ }^{1}$ School of Natural Sciences, Linnaeus University, 39182 Kalmar, Sweden \\ ${ }^{2}$ Division of Solid State Physics and The Nanometer Structure Consortium, Lund University, Box 118, S221 00 Lund, Sweden
}

Correspondence should be addressed to Alf Månsson, alf.mansson@lnu.se

Received 31 August 2011; Accepted 7 January 2012

Academic Editor: P. Bryant Chase

Copyright (C 2012 Alf Månsson et al. This is an open access article distributed under the Creative Commons Attribution License, which permits unrestricted use, distribution, and reproduction in any medium, provided the original work is properly cited.

\begin{abstract}
Self-organization phenomena are of critical importance in living organisms and of great interest to exploit in nanotechnology. Here we describe in vitro self-organization of molecular motor-propelled actin filaments, manifested as a tendency of the filaments to accumulate in high density close to topographically defined edges on nano- and microstructured surfaces. We hypothesized that this "edge-tracing" effect either (1) results from increased motor density along the guiding edges or (2) is a direct consequence of the asymmetric constraints on stochastic changes in filament sliding direction imposed by the edges. The latter hypothesis is well captured by a model explicitly defining the constraints of motility on structured surfaces in combination with Monte-Carlo simulations [cf. Nitta et al. (2006)] of filament sliding. In support of hypothesis 2 we found that the model reproduced the edge tracing effect without the need to assume increased motor density at the edges. We then used model simulations to elucidate mechanistic details. The results are discussed in relation to nanotechnological applications and future experiments to test model predictions.
\end{abstract}

\section{Introduction}

Self-organization of complex systems involves pattern or structure formation due to multiple local interactions of the participating elements when a system is left to itself. Whereas self-organization phenomena are of critical importance in biology, they are also of great interest to exploit for nanofabrication or, more dynamically, for the production of high local densities of certain molecules on a chip, for example, for biosensing. Molecular motors, predominantly myosins, and kinesins, with their respective cytoskeletal filaments, that is, actin filaments and microtubules, are instrumental for selforganization phenomena in living systems. They are thus the basis for organism motility (muscle contraction) as well as cell motility in general and transport of molecular cargoes within cells. Considerable efforts have been invested into exploiting this motion-generating machinery for nanotechnological applications [1-17]. Also self-organization phenomena of motor-propelled cytoskeletal filaments in vitro under different conditions have been considered in this context. Thus, in the presence of cross-linking molecules, stable or meta-stable filament bundles of different shapes may form [18]. In the absence of cross-linkers, on the other hand, the filaments either move collectively in partly ordered, but dynamically changing swarms [19-21], or execute random diffusion like movement [19], depending on the conditions [22]. The diffusion-like behaviour can be partly controlled by chemical and topographical micro-, and/or nanopatterns and used as a versatile method to produce actin filament gradients [19] locked to the myosin-coated surface by removal of ATP. Actin filaments are readily functionalized with various biomolecules for example, extracellular matrix proteins [19], and may serve as templates for formation of gradients of other biomolecules, useful, for example, for tissue engineering and studies of cell adhesion. Alternatively, the filaments could be derivatized with gold [23] to produce complex electrical circuits.

In previous studies [19] of actomyosin-based selforganization on topographically and chemically nanopatterned surfaces we noted that the motor-propelled actin filaments tend to accumulate along edges of the motility supporting areas. We hypothesized [19] that this effect, that 
we here denote the "edge-tracing effect," is attributed to: (1) higher local motor density at the corner between a wall and the floor of the motor supporting area or (2) reduced probability for filaments to leave their current path along the wall-floor junction because the thermal fluctuations of the free leading end of the filament are limited to one (rather than two, as on an open surface area) direction in the surface plane. The asymmetric constraints imposed by the wall on stochastic changes in filament position according to the second hypothesis may also be extended to incorporate the idea that filaments which have just moved away from the edge have an increased likelihood of hitting the edge again (cf. reasoning in [24] for a molecule close to a cell surface). This possibility is considered in some detail here.

The motility of actin filaments on micro- and nanopatterned surfaces may be simulated by Monte-Carlo approaches $[25,26]$ where the winding filament paths on open areas are characterized by a persistence length (the length along a polymer/path over which the "memory" of a tangent angle of the polymer/path is maintained. For example, the higher flexural rigidity of the polymer the longer the persistence length.) equal to that of the actin filament whereas filaments when reaching borders are forced to follow these with $100 \%$ probability. The latter is a reasonable approximation using actin filaments and walls with overhanging roofs and combined chemical and topographical patterning [10, 27].

Here we first demonstrate the edge-tracing effect in experimental data using nanostructured surfaces with actin filament guiding tracks of the type described previously [10, 27]. We then perform Monte-Carlo simulations for similar surface track geometries as in the experiments showing excellent prediction of the edge-tracing effect. This corroborates the second hypothesis above because the simulations capture the asymmetric effects of the walls on stochastic changes in motion but do not include any effects of altered motor density. The simulation results also lead to additional testable predictions that open for further in depth insight into the edge-tracing mechanism. These predictions and approaches for testing them as well as the usefulness of the edge-tracing effect for nanotechnological applications are discussed. The experimental results have previously been published in a PhD thesis [28].

\section{Methods}

2.1. Experiments. The methods for protein preparations, in vitro motility assays and recording of in vitro motility assay data have been described in detail elsewhere [10, 29-31]. The in vitro motility assays were performed at $25^{\circ} \mathrm{C}, \mathrm{pH}$ 7.4, $1 \mathrm{mM}$ MgATP, and $40 \mathrm{mM}$ ionic strength. The surfaces were preincubated with heavy meromyosin $(200 \mu \mathrm{g} / \mathrm{mL})$ for $2 \mathrm{~min}$. The methods for nanostructuring have been described $[10,27,31]$, including electron-beam lithography to produce bilayer resist channels (Figure 1) and surface derivatization of nanosized tracks and larger motility supporting areas with trimethylchlorosilane (TMCS).

2.2. Model Simulations. A Monte-Carlo simulation approach was developed on basis of previous work $[25,26]$ and im- plemented in Matlab (Mathworks, Natick, MA). In the modelling, different geometries for a motility supporting area, delimited by walls like those surrounding the tracks in Figure 1, could be simulated from an ellipse and two second degree polynomials (Figure 2 ). The polynomials either caused the right part of the motility supporting area to be concave (dashed line) or convex (full line) inwards. For $R_{\max }=R_{\min }$ (exclusively used here) and with very small $L_{1}$, the motility supporting area is approximately circular. The elliptical and polynomial parts were defined to make the border-defining function and the first derivative of this function continuous. The simulations were simplified by symmetry, allowing us to consider only the lower half of the zone in Figure 2.

In the simulations, the instantaneous angular changes in actin filament sliding direction on the open motility supporting area are assumed to be normally distributed with standard deviation

$$
\Delta \varphi=\sqrt{\frac{v_{f} \cdot \Delta t}{L_{P}}} .
$$

Here, $v_{f}$ is the sliding velocity, $\Delta t$ is the time interval $(10 \mathrm{~ms}$ unless otherwise stated) between updates in sliding direction, and $L_{P}$ is the actin filament persistence length $[25,26,32]$ that has been found to be similar to the persistence length of HMM-propelled actin filament paths in the in vitro motility assay [32]. The angular changes in sliding direction are regularly drawn from a normal distribution using inbuilt Matlab functions. If filaments, as a result of these angular updates, enter coordinates outside the defined borders of the motility supporting zone, the filaments are guided along this border [25] (synonymously denoted "wall"or "edge"). The procedures that we employ to simulate guiding along curved borders are described in greater detail in Figure 3. The procedure when a filament first reaches the wall is straightforward (Figure 3(a)) and has been illustrated previously. In contrast, the subsequent procedure, when the filament is already in contact with the wall, is not immediately obvious. We here (Figure 3(b)) take the approach that filament fluctuations are first simulated (1; with standard deviation for angular change given by (1)) as if the wall was not present. If this results in a new position at 2 in Figure 3(b), that is, beyond the border of the motility supporting region, the filament is shifted to the wall at a new position (3 in Figure 3(b)) compatible with a sliding distance $v_{f} \Delta t$ from the starting position (0). The edge-tracing with a filament in contact with the wall is interrupted if the thermal fluctuations bring the filament away from the wall, into the motility supporting area (Figure 3(c)).

\section{Results and Discussions}

3.1. Edge-Tracing Effect-General Aspects. Examples of the edge-tracing effect are shown in the experimental records in Figure 4. Here, actin filaments move along topographical borders (inset Figure 4(a)) separating TMCS areas with good motility quality from surrounding, resist-covered (PMMA), areas without motility. In Figures $4(\mathrm{a})$ and $4(\mathrm{~b})$, results are 

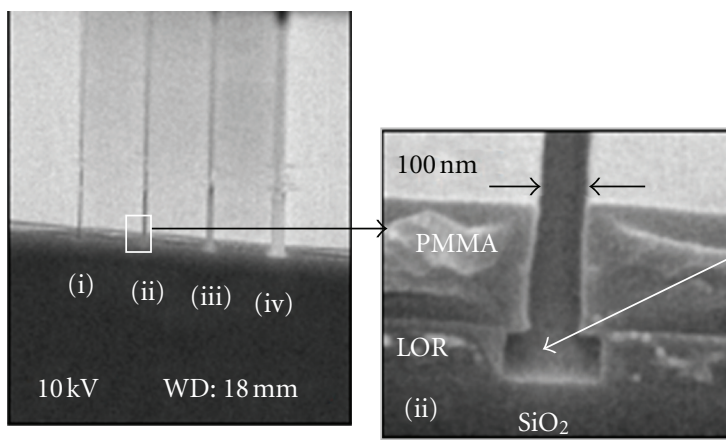

TMCS-

derivatized

channel floor

FIGURE 1: Scanning electron micrographs showing nanostructured surface produced by electron-beam lithography applied to bilayer resist layer on $\mathrm{SiO}_{2}$, consisting of lift-off resist (LOR) and polymethylmethacrylate (PMMA). Following electron-beam exposure and development, the PMMA layer was made hydrophilic by oxygen-plasma ashing followed by trimethylchlorosilane (TMCS) derivatization of the exposed $\mathrm{SiO}_{2}$. The nanoscale track illustrated here has similar walls as the motility supporting open areas illustrated below (e.g., Figure 4).

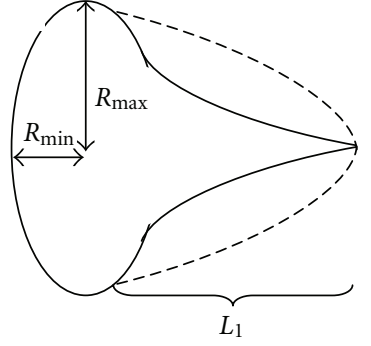

(a)

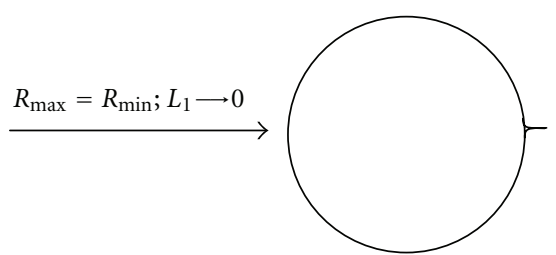

(b)

Figure 2: Geometry of motility supporting zone for simulations. (a) Generalized geometry with one part of the zone defined by an ellipse and another part defined by a second degree polynomial (e.g., filled or dashed line). Polynomials selected to be continuous with the ellipse and also with continuity of first derivative. (b) Special case with $R_{\max }=R_{\min }$ and very small value of $L_{1}$. This special case was exclusively used here.

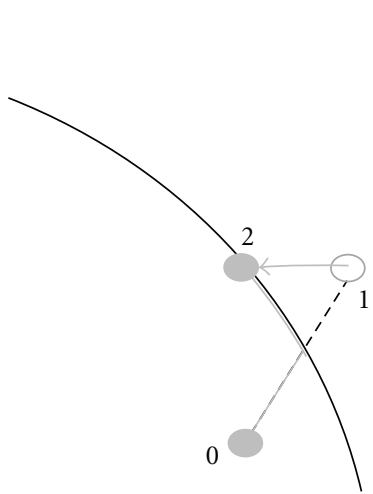

(a)

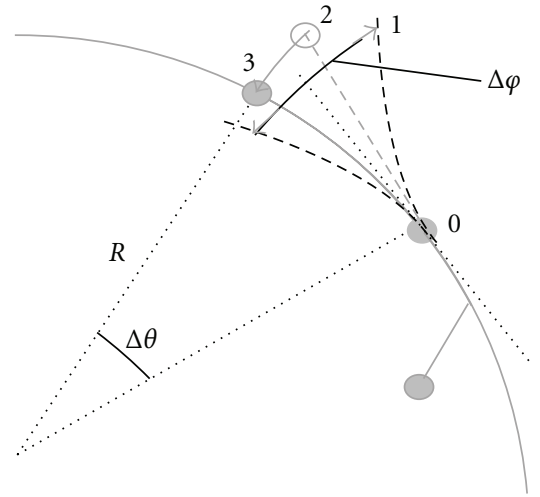

(b)

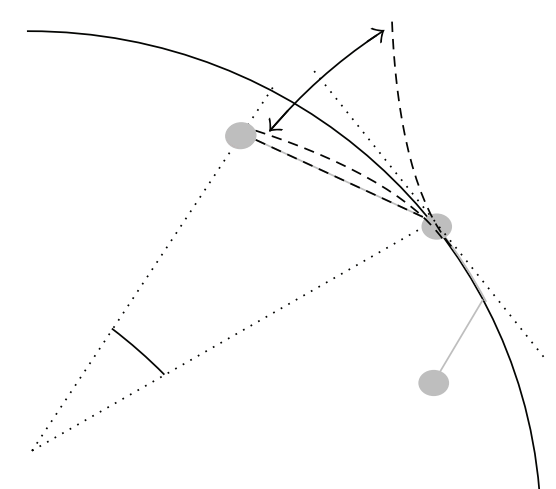

(c)

FIgURE 3: The principle of filament guiding at curved edges in the Monte-Carlo simulations. (a) Filament front (grey filled circle) reaches border from open motility supporting area. Without the border, the position 1 would be attained. In the guiding process, this is shifted to position 2 while ensuring that the total distance moved between positions 0 and 2 is equal to sliding velocity times the simulation time step $\left(v_{f} \Delta t\right)$. (b) Continued guiding along the wall. A new sliding direction is drawn from a Gaussian (with SD as in (1)) under the assumption that the wall is not present. Even if the new position will be outside the wall, the filament front is first moved a distance $v_{f} \Delta t$ to that position (2). The filament is then shifted to position 3 on the border in a process that maintains the sliding distance, $v_{f} \Delta t$, between positions 0 and 3 . (c) The process if the random update in sliding direction moves the filament away from the border. Note, that the ratio $v_{f} \Delta t / R$ is unrealistically large in this example for enhanced clarity in description of the guiding process. 


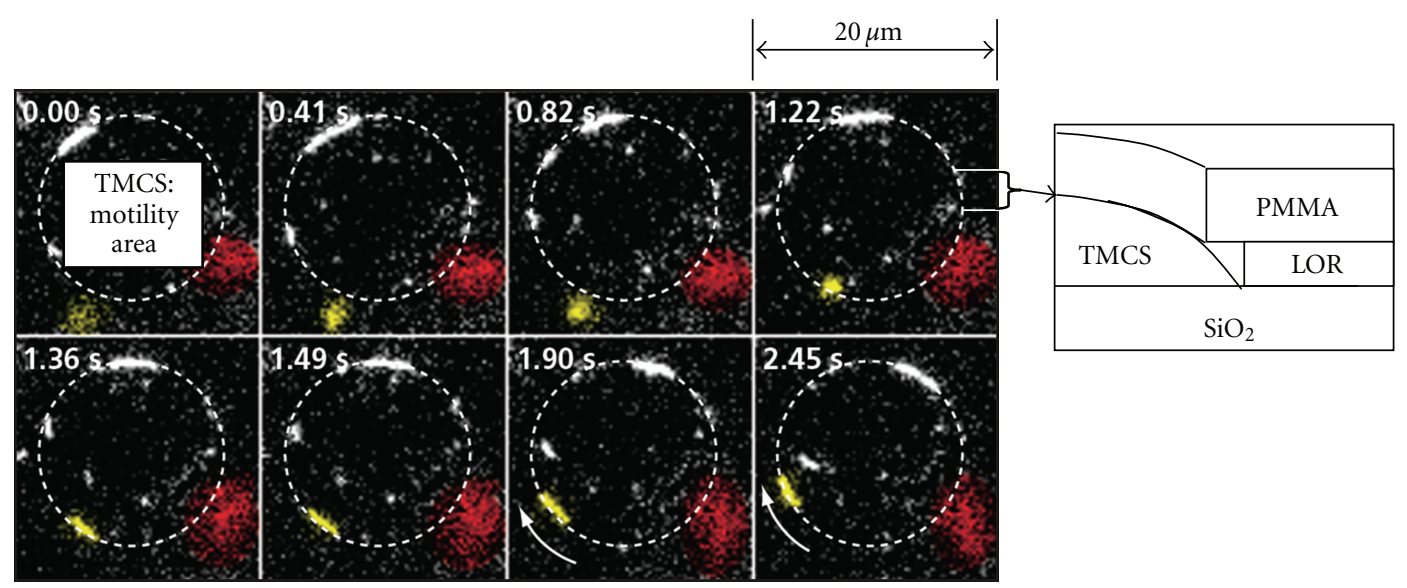

(a)

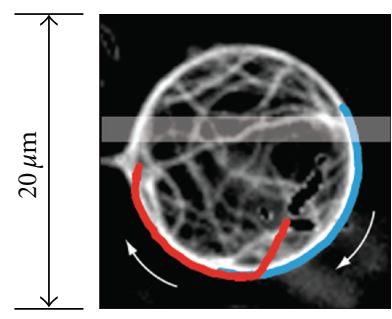

(b)

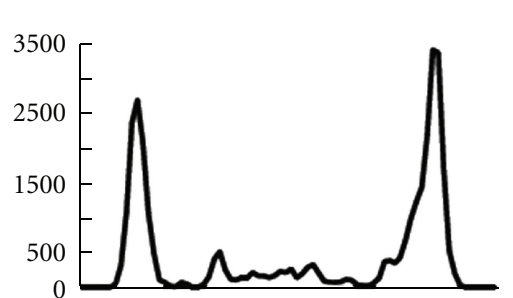

(c)

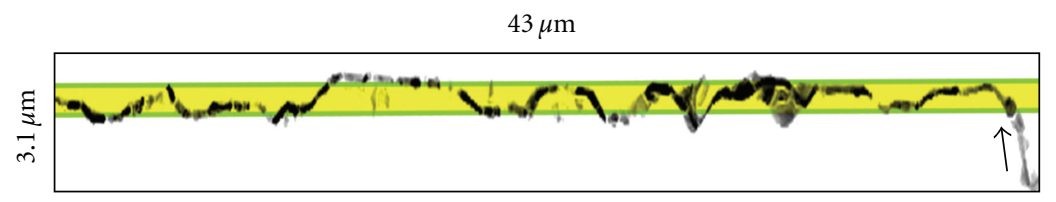

(d)

FIGURE 4: Experimental demonstration of the edge-tracing effect. (a) A time sequence of fluorescence micrographs showing actin filaments moving on a disk-shaped motility supporting TMCS surface surrounded by hydrophilic and negatively charged PMMA (see inset with schematic illustration of topography between full white lines in rightmost micrograph in first row). The lack of actin binding on PMMA indicated by red and yellow filaments floating in solution. Note that several of the HMM-propelled actin filaments on the disk-shaped TMCS-derivatized region move along the border of the motility supporting area. (b) Fluorescence micrograph integrated over $60 \mathrm{~s}$ to show sliding trajectories of 8 motile filaments (4 stationary filaments) sliding at velocity of about $3 \mu \mathrm{m} / \mathrm{s}$. (c) Intensity profile indicating the density of actin filaments along the gray area in (b). (d) Time-integrated and color-enhanced fluorescence micrograph illustrating path of a filament (length $3.5 \mu \mathrm{m}$ ) entering a channel (yellow-green area) from an open zone at the arrow. The image is not to scale but magnified $2 x$ vertically in order to more clearly illustrate the lateral position of the filament inside the channel. Green: approximate bottom channel borders (width $700 \mathrm{~nm}$; LOR walls). Yellow: approximate top channel position (width $600 \mathrm{~nm}$; with PMMA walls) for the type of channels illustrated in Figure 1. Figure 4(d) reproduced from [27] (DOI: 10.1088/0957-4484/16/6/014) with permission from Institute of Physics.

illustrated for a nearly circular disk-shaped motility supporting area, and in Figure 4(d) data are shown for filaments sliding along a $700 \mathrm{~nm}$ wide channel of the type illustrated in Figure 1. A simulation of the experimental situation in Figure 4(b) is illustrated in Figures 5(a) and 5(b) using similar conditions as in the experiment. That is, in Figure 5(a), about 10 filaments are assumed to be deposited randomly on the diskshaped motility supporting surface followed by simulation of the filament paths for $60 \mathrm{~s}$ using similar velocity $(\sim 3 \mu \mathrm{m} / \mathrm{s})$ as in the experiments. It is clear that the simulated behaviour is similar to that seen in the experiment in Figure 4(a) with preferential accumulation of filaments close to borders. This is also emphasized in Figure 5(b) where the radial density distribution of the number of filaments is shown, corresponding to half the experimental intensity profile in Figure 4(c). We also simulated heavy meromyosin-propelled actin filament sliding along straight tracks similar to those in Figures 1 and 4(d). Typical results are illustrated for track widths between 0.2 and $1.2 \mu \mathrm{m}$ in Figures $5(\mathrm{c})-5(\mathrm{e})$. It can be seen that, in accordance with the experimental result in Figure 4(d), the simulated paths exhibit an obvious edge-tracing effect. Moreover, after losing contact with an edge, the simulated filament paths, like the experimental one, either shifted rapidly back to the same wall or moved swiftly to the other wall (corresponding to reflection in the top border in the records in Figures 5(c)-5(e)). 


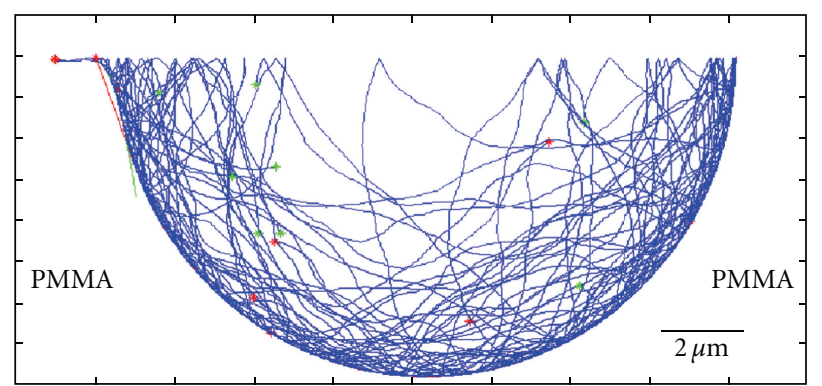

(a)

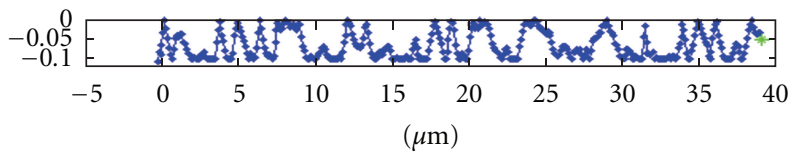

(c)

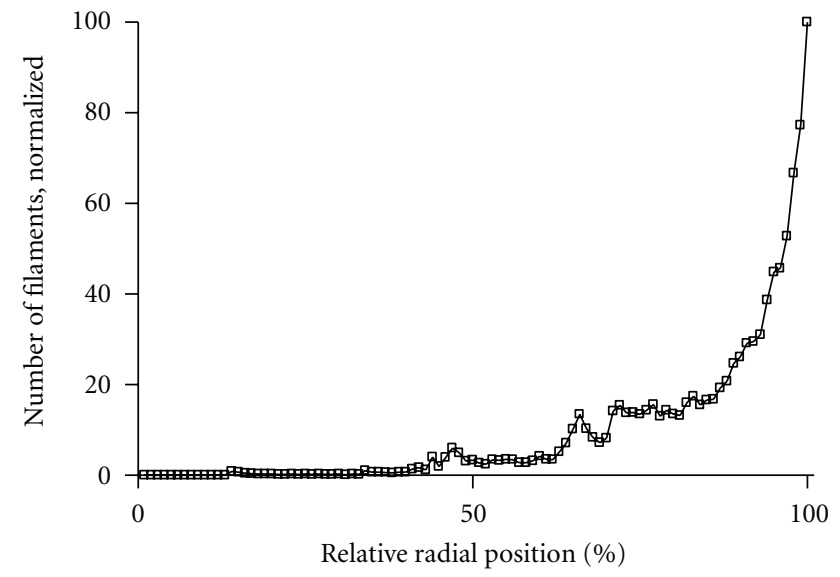

(b)

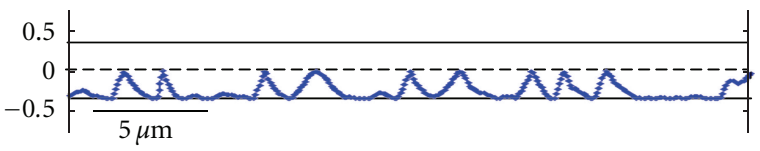

(d)

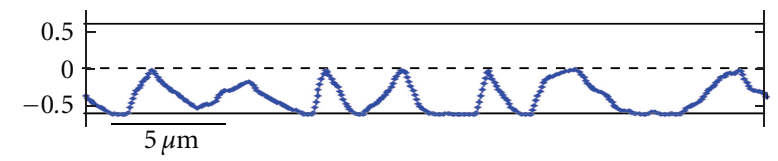

(e)

FiguRE 5: Monte-Carlo simulations of actin filament paths for the experimental situation in Figures 4(b) and 4(c) with 8 motile filaments (10 filaments in the simulations for practical reasons), a velocity of $3 \mu \mathrm{m} / \mathrm{s}$, and a total integration time of $60 \mathrm{~s}$. (a) Simulated filament paths (blue) with starting points (green) and end-points (red). The motility supporting area is indicated by weak red (circular part) and green (to the far left; polynomial part) lines. For symmetry reasons, the simulations were limited to a half-circular shape which simplified the algorithm. Thus, filament paths are reflected in the top border of the simulated area, corresponding to their motility with unchanged sliding direction past this border. (b) The radial distribution of filament positions accumulated during the entire simulation period. Here, 0 corresponds to the centre of the circular motility supporting zone, and 100\% corresponds to the border with the LOR/PMMA-area. (c) Simulation of sliding along $200 \mathrm{~nm}$ wide track similar to that in Figure 1. Simulation limited to lower half of the track with reflection of filament paths in track midline (top of diagram). Thus, simulated filament paths at bottom of figure correspond either to motility at the lower or top edge of the track. (d) Simulation of sliding along $700 \mathrm{~nm}$ wide track (delimited by upper and lower full lines) as in Figure 4(d). Simulation only for lower half of track with reflection in midline (dashed line) as in Figure 5(c). (e) Simulation as in Figure 5(d) but track width increased to $1.2 \mu \mathrm{m}$.

3.2. Edge-Tracing Radius of Motility Supporting Region versus Filament Persistence Length. For further elucidation of the mechanisms underlying the edge-tracing effects we next turned to simulation of filament paths on motility supporting areas of different radii. The results for 50-100 simulated filaments for 3 different radii of the motility supporting area are illustrated in Figure 6(a). It is clear that the spatial variation of the number of filaments within a given motility supporting area is similar to that observed with considerably fewer filaments in Figure 5. However, it can also be seen more clearly in Figure 6(a) that the edge-tracing effect is superimposed on a linear increase in the number of filaments with radius. This linear increase is trivially expected for randomly distributed objects on a circular area due to increased circumference with increased radial distance. The edge-tracing effect (the filament number at edges in excess of that expected from the linear increase with radius) in simulated data can be seen for example, for a radius $(R)>15 \mu \mathrm{m}$ for a motility supporting zone of $20 \mu \mathrm{m}$ radius in Figure 6(a) (blue lines). The simulated edge-tracing effect, however, becomes more prominent with reduced radius of the motility supporting area (Figures $6(\mathrm{a})-6(\mathrm{c})$ ) as well as with increased filament persistence length (Figure 6(c)); that is, the edge-tracing effect increased with a reduction of the $R / L_{P}$ ratio (Figure $6(\mathrm{c})$ ). In contrast, altered sliding velocity did not influence the edge-tracing effect in the simulations (Figure 6(d)). The edge-tracing effect was fully developed about $5 \mathrm{~s}$ after onset of the simulations for a $20 \mu \mathrm{m}$ radius motility supporting zone, a velocity of $2.5 \mu \mathrm{m} / \mathrm{s}$ and a persistence length of $10 \mu \mathrm{m}$. In all other cases $(R<$ $20 \mu \mathrm{m}, v_{f}>2.5 \mu \mathrm{m} / \mathrm{s}$ and $L_{P} \geq 10 \mu \mathrm{m}$ ), the effect was fully developed within $2.5 \mathrm{~s}$. 


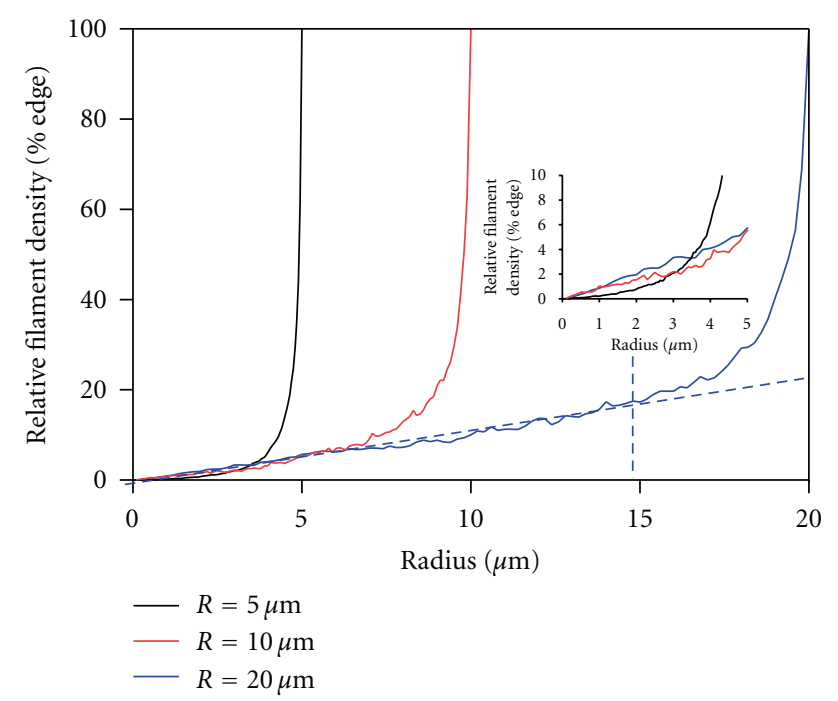

(a)

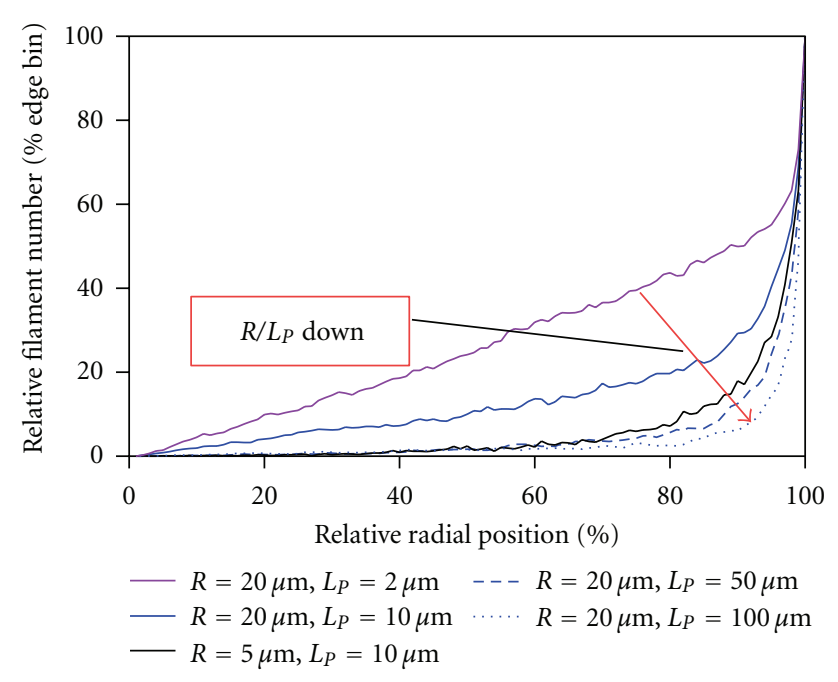

(c)

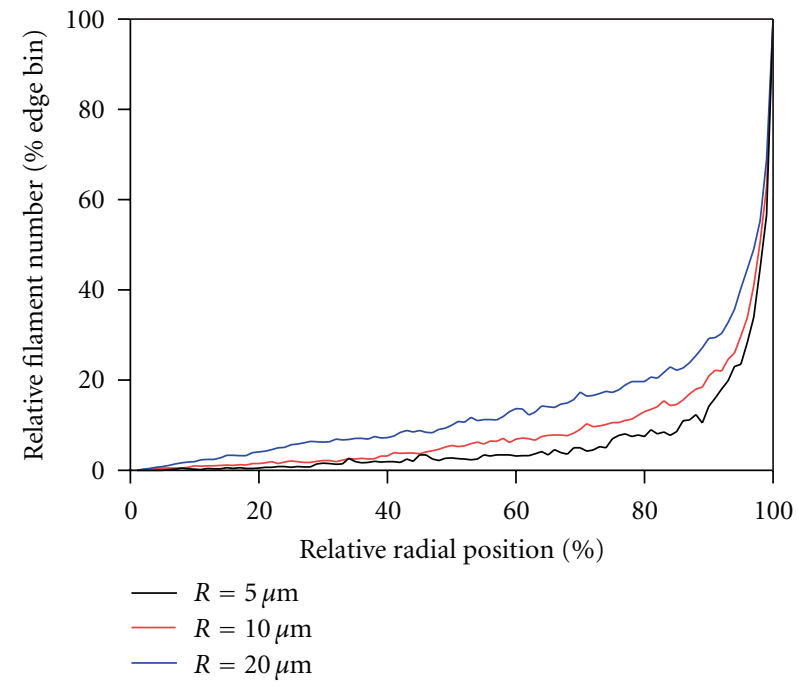

(b)

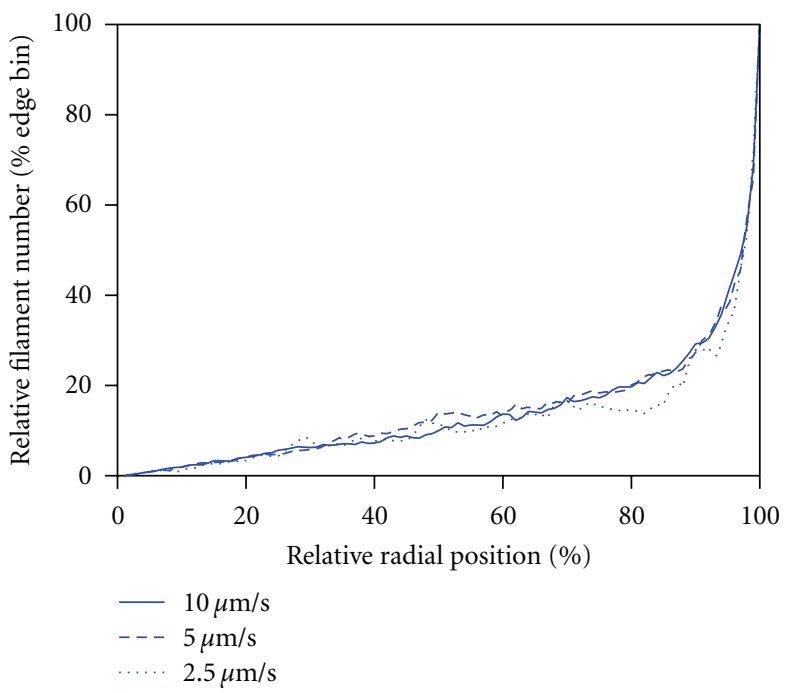

(d)

FiguRE 6: Radial filament distributions simulated for motor-propelled filaments. (a) The effect of radius $(R)$ of motility supporting region on the radial distribution of filaments. $L_{p}=10 \mu \mathrm{m}, V_{f}=10 \mu \mathrm{m} / \mathrm{s}, \Delta t=0.01 \mathrm{~s}$. Note, a small linear increase in filament number with increased radius related to the increase of circumference. Edge-tracing effect corresponds to steep increase (in excess of the linear change) in number of filaments at periphery, for example, to the right of the dashed blue line for $R=20 \mu \mathrm{m}$. Inset: part of diagram at the origin shown in greater detail. (b) The data in A, replotted with the radial position normalized to the radius of the motility supporting region. (c) Radial filament distributions for different $L_{P}-R$ combinations $\left(v_{f}=10 \mu \mathrm{m} / \mathrm{s}\right.$ and $\left.\Delta t=0.01 \mathrm{~s}\right)$. Note, increased edge-tracing with reduced $R / L_{P}$ ratio. That is, under these conditions, the edge peak in filament number is shifted outwards and has increased amplitude relative to the expected linear increase for all radial distances. (d) Radial filament distributions for different sliding velocities $\left(R=20 \mu \mathrm{m}, L_{P}=10 \mu \mathrm{m}\right.$ and $\left.\Delta t=0.01 \mathrm{~s}\right)$. Distributions obtained during time periods over which no apparent time dependence of the distributions was noted. The unrealistically low value of $L_{P}=2 \mu \mathrm{m}$ (compared to actin filaments and microtubules) was included in (c) to more clearly illustrate the effect of altered $R / L_{P}$ ratio.

3.3. Hypothesis 2-Detailed Mechanisms Underlying EdgeTracing. Before interpreting the simulation results in relation to experimental data it is important to consider what is actually happening in the simulations when a filament is guided at the edge. As described in Figure 3, the free leading end of the filament is assumed to execute thermal fluctuations governed by a Gaussian distribution with standard deviation as in (1). For a straight (corresponding to circular motility zone of infinite radius) rather than curved wall, this would mean that in 50\% of the trials (corresponding to capture by a new myosin head) the filament will move away from the wall. On this basis the filament would, on average, stay in contact with the wall for up to $100 \mathrm{~nm}$ before escaping. This may be inferred from the average value (1 failure before escape) for a corresponding geometric distribution and the average distance $(\sim 40-100 \mathrm{~nm}$; $[32,33])$ between sequential captures of the actin filament tip on myosin heads. Furthermore, several events with edge tracing 
for $>100 \mathrm{~nm}$ would be expected. For instance, on the assumption of $100 \mathrm{~nm}$ distance between subsequent capture of the filament tip on myosin heads, the probability is $1-$ $\sum_{i=1}^{3} 0.5^{i}=12.5 \%$ that filaments stay in contact with straight edges for $>300 \mathrm{~nm}$ before escaping. The occurrence of such events can be seen in the simulated data in Figures 5(c)5 (e) (corresponding to $>4$ simulated points) and also appear to exist in the experimental data in Figure 4(d) (within the experimental uncertainty).

From Figure 3 it can be readily inferred that a concave wall towards the motility supporting area, as in Figures 4(a), $4(\mathrm{~b})$, and $5(\mathrm{a})$, would reduce the probability of escape from the edge. Thus, for a filament that moves a distance $v_{f} \Delta t$ along the edge, the edge tangent will rotate by an angle $\Delta \theta$, given by (cf. Figure 3(b))

$$
\Delta \theta R=v_{f} \Delta t .
$$

This rotation reduces the probability for the thermal oscillations of the actin filament tip to bring the tip to a position away from the wall. This can be interpreted as an elastic binding energy $(\Delta U)$ that tends to hold the free leading end of a filament in contact with edges with a concave curvature towards the motility supporting region. The energy can be expressed as

$$
\Delta U=-\frac{1}{2 R^{2}} v_{f} \Delta t L_{P} k_{B} T,
$$

where $k_{B} T$ is the Bolzmann factor. In contrast to concave edges (e.g., Figure 3 ) inwards convex edges (not further considered below) would have a tendency to "repel" the filament with a "repulsion energy," $\Delta U$, with the same magnitude as in (3) but with a positive sign.

For both concave and convex edges, $\Delta U$ is significant compared to thermal energy only if $v_{f} \Delta t L_{p} \geq 2 R^{2}$. Particularly, the effect of elastic binding forces is negligible for all values of $\mathrm{R}$ studied here $(R \geq 5 \mu \mathrm{m})$, assuming realistic distances between successive encounters of actin filaments with myosin heads in the in vitro motility assay $(\leq 100 \mathrm{~nm}$; see above) and an actin filament persistence length of $\sim 10 \mu \mathrm{m}$; [32]. The situation would be different for considerably increased values of $L_{p}$, reduced values of $R$, and/or increased distance $\left(v_{f} \Delta t\right)$ between successive motors, as if using stiff microtubules propelled by processive kinesin motors at low surface density.

It is important to note that edge tracing was observed in the Monte-Carlo simulations without the assumption of any other additional binding energy, for example, assuming higher myosin head densities to keep the filaments at edges. Under these conditions, the Monte-Carlo simulations, in combination with the asymmetric constraints imposed by borders (Figure 3), capture the essential elements of the second hypothesis put forward in Introduction. The faithful reproduction of the edge tracing in the simulations therefore supports this hypothesis.

Above, we have considered two mechanisms for the edgetracing effect that may be viewed as two versions of hypothesis 2 because asymmetric constraints on changes in sliding direction at edges are central in both mechanisms. These mechanisms are (i) the $50 \%$ probability of a filament to stay at a straight edge for each new capture by a myosin motor and (ii) the effect of an "elastic binding energy" at curved edges. However, despite the fact that we have shown the latter effect to be negligible under the conditions considered here the simulated filament paths remain close to the edges for considerably longer average distances than predicted by mechanism (i) $(\leq 100 \mathrm{~nm})$. Therefore, there must be an additional mechanistically different version of hypothesis 2 (mechanism (iii)) that contributes to the edge-tracing effect. This is also suggested by the fact that the edge tracing effect was considerably enhanced by reduced $R$ and increased $L_{P}$ in the simulations despite negligible changes in $\Delta U$ within the ranges of $R$ and $L_{P}$ studied. As a basis for mechanism (iii), we consider the fact that filaments which have once encountered the border will tend to be rectified in their sliding along that border. Further, the memory of this sliding direction is maintained for path lengths short compared to the persistence length even if the filaments do not stay in direct contact with the edge. More insight into the above mechanisms (particularly mechanism (iii)) are gained in Figure 7. Here, we simulate an ensemble of filament paths all with initial sliding direction along the tangent of a circle of $20 \mu \mathrm{m}$ radius. It can be seen (Figure 7) that the simulations predict that $\sim 50 \%$ of the filaments would be outside the circle after a few $\mathrm{nm}$ of sliding. This may be thought to correspond to mechanism (i) above. Thus, if there had been an edge along the drawn circle in Figure 7 and if the motility supporting region had been inside the circle (on top in Figure 7), these filaments would have remained sliding along that edge. Also most of those simulated filaments in Figure 7 that did not cross the circular line (towards the bottom of the figure) tend to move close to it as a result of their initial rectification along the tangent and a persistence length of $10 \mu \mathrm{m}$. As a consequence, within about $10 \mu \mathrm{m}$ of sliding, $90 \%$ of all filaments, would again have reached and crossed the circular line (downwards in Figure 7). This means that, in the presence of an edge, the filaments would again be guided along this. It is straightforward to realize that this effect would increase when $L_{P}$ increases relative to the radius of curvature of the zone with motility. Thus, filaments with larger $L_{P}$ that have left the wall would "have a longer memory" of the sliding direction, thereby more rapidly hitting the wall again. It is illuminating to consider the extreme (but unrealistic) situation that the radius of the motility supporting zone would go to zero. Under these conditions the probability would go to 1 that filaments escaping from the edge immediately reach the edge again. The mentioned effects were seen in the simulations in Figure 6 as considerably increased edge-tracing effect, with larger fraction of the filaments closer to the edge, for reduced ratio of $R / L_{P}$ (Figure 6(c)).

The edge-tracing effect was superimposed on the linear increase in filament number (cf. Figure 6(a)) expected with increased radius of the motility supporting region for diffusion like filament transport [19]. However, if $R$ is small compared to $L_{P}$, the influence of ballistic transport is substantial for most filaments in the motility supporting region, consistent with our finding that the edge-tracing effect 


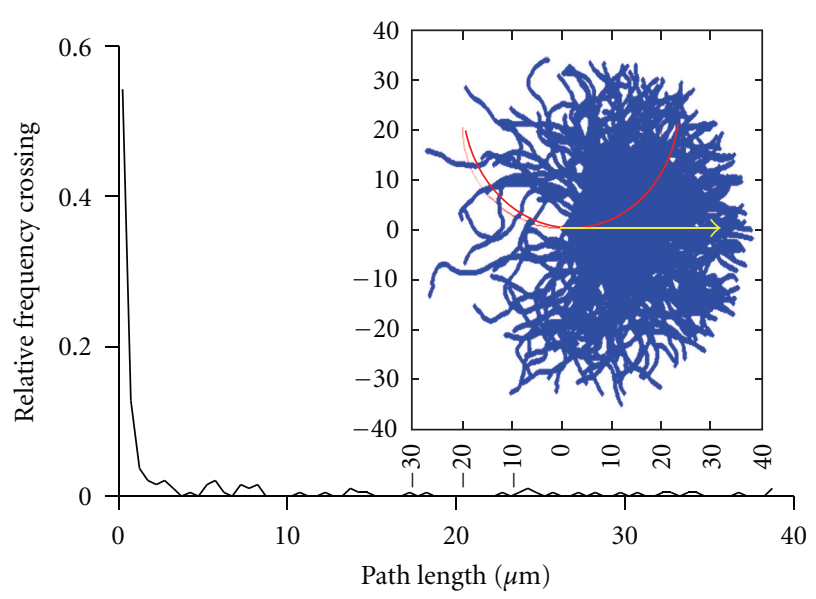

(a)

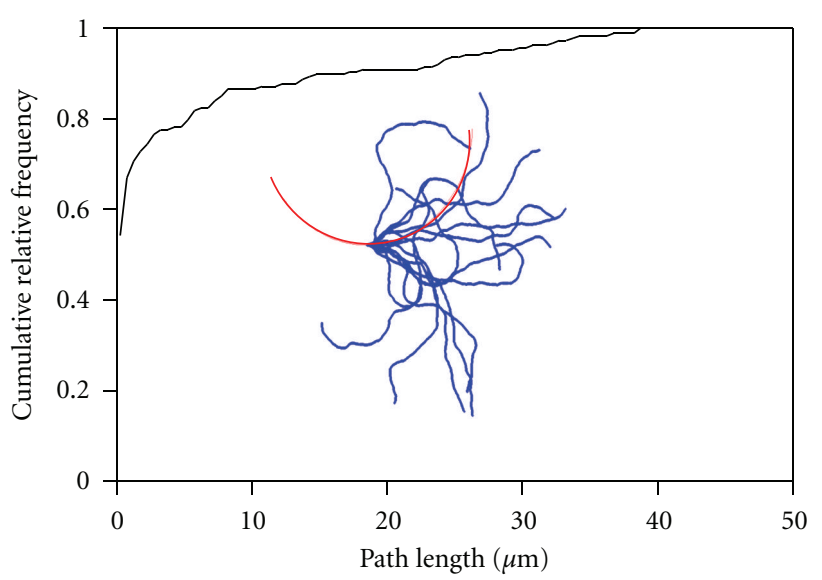

(b)

FIGURE 7: Monte-Carlo simulation of HMM-propelled actin filament sliding $\left(L_{P}=10 \mu \mathrm{m}\right)$ without guiding but with the same initial position and sliding direction for all filaments. (a) Distribution of sliding path lengths from onset of motility until the filament, for the first time, moves below red circle arc in inset. Inset: all 200 simulated filament paths starting on the red circular border at the starting point of the arrow. The direction of the latter represents the initial sliding direction. (b) Cumulative frequency distribution corresponding to frequency distribution in (a). Inset: a limited ensemble of 10 simulated filament paths clearly illustrating that also filaments that have moved toward the circle centre tend to rapidly reach and cross the circular border.

(see above) is fully developed within seconds. Clearly, in agreement with the discussions above, ballistic transport in a direction governed by the tangent along the edge, completely dominates transport for those filaments that have just left this edge.

It should be clear from the above discussion that the second hypothesis in the Introduction accounts well for the edge-tracing effect without the need to assume higher HMM densities close to edges. We have also shown that this second hypothesis, when considered in greater detail, may be subdivided into three different mechanisms ((i)-(iii) above).

The Monte-Carlo simulations predict that a large fraction of the "edge-tracing" filaments are not actually in contact with but rather very close to the edge. This is broadly consistent with our experimental results. However, it is important to note that these results were included here as a background to the simulations and not for critical testing of model predictions. For such testing, future dedicated experiments would be required where particular attention should be directed to the spatial resolution in the experimental records.

However, in spite of some experimental uncertainties, it is of interest to note that the experimental results for sliding along straight tracks (Figure 4(d)) and the simulations in Figures 5(c)-5(e) seem to be reasonably consistent with each other (within the spatial resolution) both with regard to the number of edge-tracing events and their duration. Future dedicated experiments to test the mechanisms above should include studies of guiding along straight borders (with mechanism (i) dominating) as well as studies of guiding along borders with varying curvature. The latter type of studies would particularly probe the importance of mechanism (iii) unless filaments of long persistence length (e.g., microtubules or actin filament bundles) are used in which case mechanism ii may also be important.
3.4. Perspectives and Conclusions. The present study has shown that the edge-tracing effect can be accounted for by asymmetric constraints on stochastic changes in filament sliding direction along edges (hypothesis 2 in the Introduction). Particularly at edges that are concave towards the motility supporting region, constraints seem to include an important component attributed to temporary rectification of filament sliding that tends to keep the filaments close to the edge for distances short compared to the filament persistence length. We have proposed experiments above to gain further insight into the three mechanisms encompassed within hypothesis 2 and to test for possible contribution of other factors. The increased insight into mechanisms behind the edge tracing effect may be useful for enhanced concentration of motor-transported cargoes at edges (cf. [13]). The results would also guide the production of gradients of actin filaments of certain shapes, thereby adding to the versatility of previous approaches [19]. One may also expect that edgetracing could increase the versatility and spatial resolution of surface imaging methods based on molecular motor driven filament transport [34]. Finally, the results may be useful for faster and more efficient feeding of filaments into tracks of nanotransportation devices (see Supporting Information; $[10,27,35])$.

\section{Disclosure}

Månsson is a cofounder, coowner, and CEO of the start-up company ActoSense Biotech $\mathrm{AB}$ (Kalmar, Sweden) aiming to develop diagnostic devices based on the aggregation of cytoskeletal elements, particularly actin filaments, in solution. Moreover, A. Mansson holds two Swedish patents in this field and application for one of these patents (about aggregation of actin filaments by analyte molecules) has also been filed in the US and Europe. 


\section{Acknowledgments}

This work was funded by the European commission (FP7) under the contract MONAD (NMP4-SL-2009-228971), The Swedish Research Council (Project no. 621-2010-5146), The Carl Trygger Foundation, The Swedish Foundation for Strategic Research, The Knowledge Foundation (KK-stiftelsen), The Faculty of Natural Sciences and Engineering at Linnaeus University, and The Nanometer Consortium at Lund University.

\section{References}

[1] H. Suzuki, A. Yamada, K. Oiwa, H. Nakayama, and S. Mashiko, "Control of actin moving trajectory by patterned poly(methylmethacrylate) tracks," Biophysical Journal, vol. 72, no. 5, pp. 1997-2001, 1997.

[2] D. V. Nicolau, H. Suzuki, S. Mashiko, T. Taguchi, and S. Yoshikawa, "Actin motion on microlithographically functionalized myosin surfaces and tracks," Biophysical Journal, vol. 77, no. 2, pp. 1126-1134, 1999.

[3] H. Hess, J. Clemmens, D. Qin, J. Howard, and V. Vogel, "Lightcontrolled molecular shuttles made from motor proteins carrying cargo on engineered surfaces," Nano Letters, vol. 1, no. 5, pp. 235-239, 2001.

[4] Y. Hiratsuka, T. Tada, K. Oiwa, T. Kanayama, and T. Q. P. Uyeda, "Controlling the direction of kinesin-driven microtubule movements along microlithographic tracks," Biophysical Journal, vol. 81, no. 3, pp. 1555-1561, 2001.

[5] R. Bunk, J. Klinth, J. Rosengren et al., "Towards a "nanotraffic" system powered by molecular motors," Microelectronic Engineering, vol. 67-68, pp. 899-904, 2003.

[6] J. A. Jaber, P.B. Chase, and J.B. Schlenoff, "Actomyosin-driven motility on patterned polyelectrolyte mono- and multilayers," Nano Letters, vol. 3, no. 11, pp. 1505-1509, 2003.

[7] H. Hess, G. D. Bachand, and V. Vogel, "Powering nanodevices with biomolecular motors," Chemistry-A European Journal, vol. 10, no. 9, pp. 2110-2116, 2004.

[8] P. Manandhar, L. Huang, J. R. Grubich, J. W. Hutchinson, P. B. Chase, and S. Hong, "Highly selective directed assembly of functional actomyosin on Au surfaces," Langmuir, vol. 21, no. 8, pp. 3213-3216, 2005.

[9] G. D. Bachand, S. B. Rivera, A. Carroll-Portillo, H. Hess, and M. Bachand, "Active capture and transport of virus particles using a biomolecular motor-driven, nanoscale antibody sandwich assay," Small, vol. 2, no. 3, pp. 381-385, 2006.

[10] M. Sundberg, R. Bunk, N. Albet-Torres et al., "Actin filament guidance on a chip: toward high-throughput assays and labon-a-chip applications," Langmuir, vol. 22, no. 17, pp. 72867295, 2006.

[11] M. G. L. Van Den Heuvel and C. Dekker, "Motor proteins at work for nanotechnology," Science, vol. 317, no. 5836, pp. 333336, 2007.

[12] A. Goel and V. Vogel, "Harnessing biological motors to engineer systems for nanoscale transport and assembly," Nature Nanotechnology, vol. 3, no. 8, pp. 465-475, 2008.

[13] T. Fischer, A. Agarwal, and H. Hess, "A smart dust biosensor powered by kinesin motors," Nature Nanotechnology, vol. 4, no. 3, pp. 162-166, 2009.

[14] L. Rios and G. D. Bachand, "Multiplex transport and detection of cytokines using kinesin-driven molecular shuttles," Lab on a Chip, vol. 9, no. 7, pp. 1005-1010, 2009.
[15] A. Agarwal and H. Hess, "Biomolecular motors at the intersection of nanotechnology and polymer science," Progress in Polymer Science, vol. 35, no. 1-2, pp. 252-277, 2010.

[16] T. Korten, A. Månsson, and S. Diez, "Towards the application of cytoskeletal motor proteins in molecular detection and diagnostic devices," Current Opinion in Biotechnology, vol. 21, no. 4, pp. 477-488, 2010.

[17] H. Takatsuki, K. M. Rice, S. Asano et al., "Utilization of myosin and actin bundles for the transport of molecular cargo," Small, vol. 6, no. 3, pp. 452-457, 2010.

[18] H. Hess, J. Clemmens, C. Brunner et al., "Molecular self-assembly of "nanowires" and "nanospools" using active transport," Nano Letters, vol. 5, no. 4, pp. 629-633, 2005.

[19] P. G. Vikhorev, N. N. Vikhoreva, M. Sundberg et al., "Diffusion dynamics of motor-driven transport: gradient production and self-organization of surfaces," Langmuir, vol. 24, no. 23, pp. 13509-13517, 2008.

[20] T. Butt, T. Mufti, A. Humayun et al., "Myosin motors drive long range alignment of actin filaments," The Journal of Biological Chemistry, vol. 285, no. 7, pp. 4964-4974, 2010.

[21] V. Schaller, C. Weber, C. Semmrich, E. Frey, and A. R. Bausch, "Polar patterns of driven filaments," Nature, vol. 467, no. 7311, pp. 73-77, 2010.

[22] P. Kraikivski, R. Lipowsky, and J. Kierfeld, "Enhanced ordering of interacting filaments by molecular motors," Physical Review Letters, vol. 96, no. 25, Article ID 258103, 4 pages, 2006.

[23] F. Patolsky, Y. Weizmann, and I. Willner, "Actin-based metallic nanowires as bio-nanotransporters," Nature Materials, vol. 3, no. 10, pp. 692-695, 2004.

[24] H. C. Berg and E. M. Purcell, "Physics of chemoreception," Biophysical Journal, vol. 20, no. 2, pp. 193-219, 1977.

[25] T. Nitta, A. Tanahashi, M. Hirano, and H. Hess, "Simulating molecular shuttle movements: towards computer-aided design of nanoscale transport systems," Lab on a Chip, vol. 6, no. 7, pp. 881-885, 2006.

[26] T. Nitta, A. Tanahashi, Y. Obara et al., "Comparing guiding track requirements for myosin-and kinesin-powered molecular shuttles," Nano Letters, vol. 8, no. 8, pp. 2305-2309, 2008.

[27] R. Bunk, M. Sundberg, A. Månsson et al., "Guiding motorpropelled molecules with nanoscale precision through silanized bi-channel structures," Nanotechnology, vol. 16, no. 6, pp. 710-717, 2005.

[28] R. Bunk, Creation of a Nanometer-Scale Toolbox for Molecular Motor Transport-Circuits, Lund University, 2005.

[29] J. Klinth, A. Arner, and A. Månsson, "Cardiotonic bipyridine amrinone slows myosin-induced actin filament sliding at saturating [MgATP]," Journal of Muscle Research and Cell Motility, vol. 24, no. 1, pp. 15-32, 2003.

[30] A. Månsson and S. Tågerud, "Multivariate statistics in analysis of data from the in vitro motility assay," Analytical Biochemistry, vol. 314, no. 2, pp. 281-293, 2003.

[31] M. Sundberg, M. Balaz, R. Bunk et al., "Selective spatial localization of actomyosin motor function by chemical surface patterning," Langmuir, vol. 22, no. 17, pp. 7302-7312, 2006.

[32] P. G. Vikhorev, N. N. Vikhoreva, and A. Månsson, "Bending flexibility of actin filaments during motor-induced sliding," Biophysical Journal, vol. 95, no. 12, pp. 5809-5819, 2008.

[33] Y. Harada, K. Sakurada, T. Aoki, D. D. Thomas, and T. Yanagida, "Mechanochemical coupling in actomyosin energy transduction studied by in vitro movement assay," Journal of Molecular Biology, vol. 216, no. 1, pp. 49-68, 1990. 
[34] H. Hess, J. Clemmens, J. Howard, and V. Vogel, "Surface imaging by self-propelled nanoscale probes," Nano Letters, vol. 2, no. 2, pp. 113-116, 2002.

[35] T. Kim, L. J. Cheng, M. T. Kao, E. F. Hasselbrink, L. Guo, and E. Meyhöfer, "Biomolecular motor-driven molecular sorter," Lab on a Chip, vol. 9, no. 9, pp. 1282-1285, 2009. 

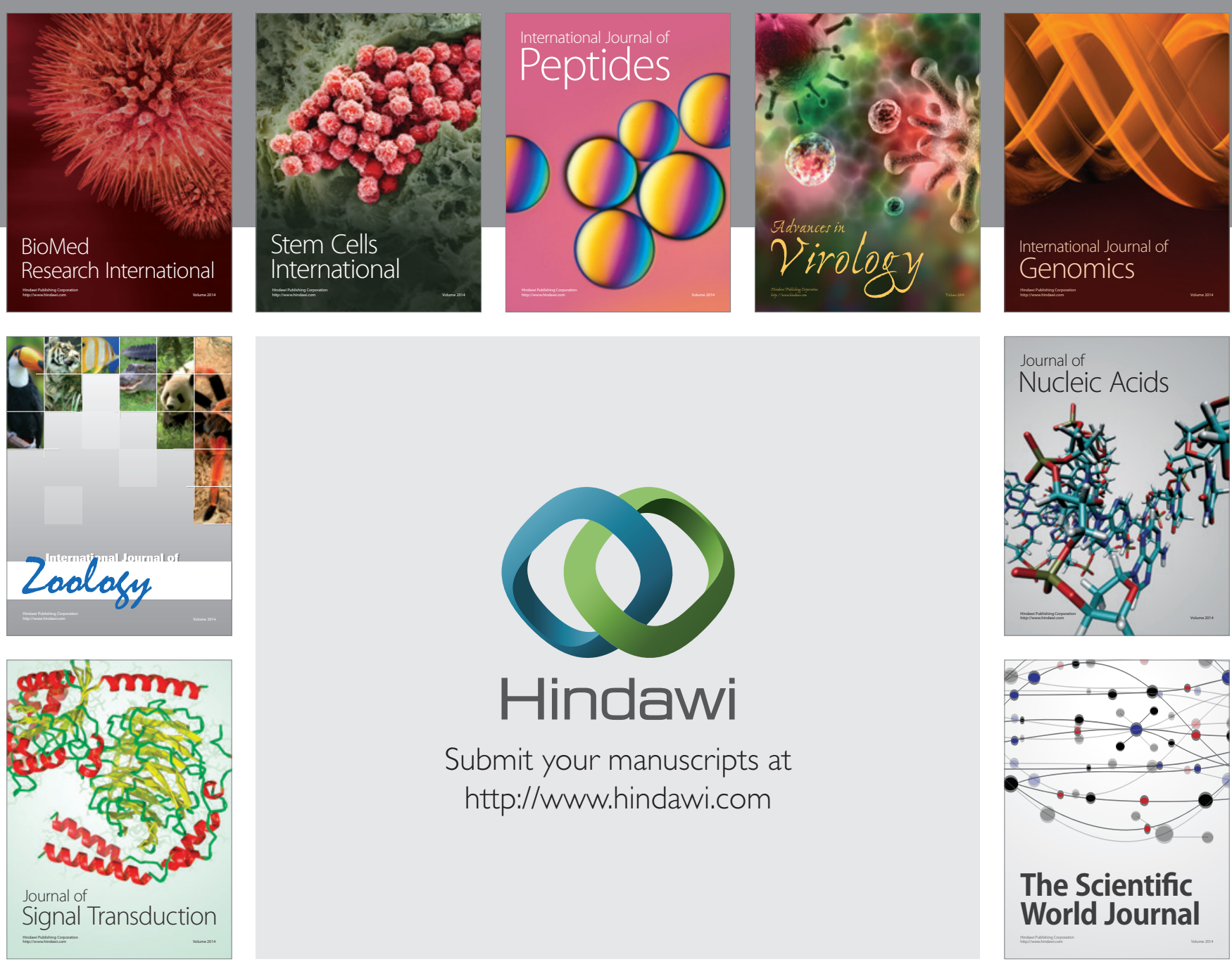

Submit your manuscripts at

http://www.hindawi.com
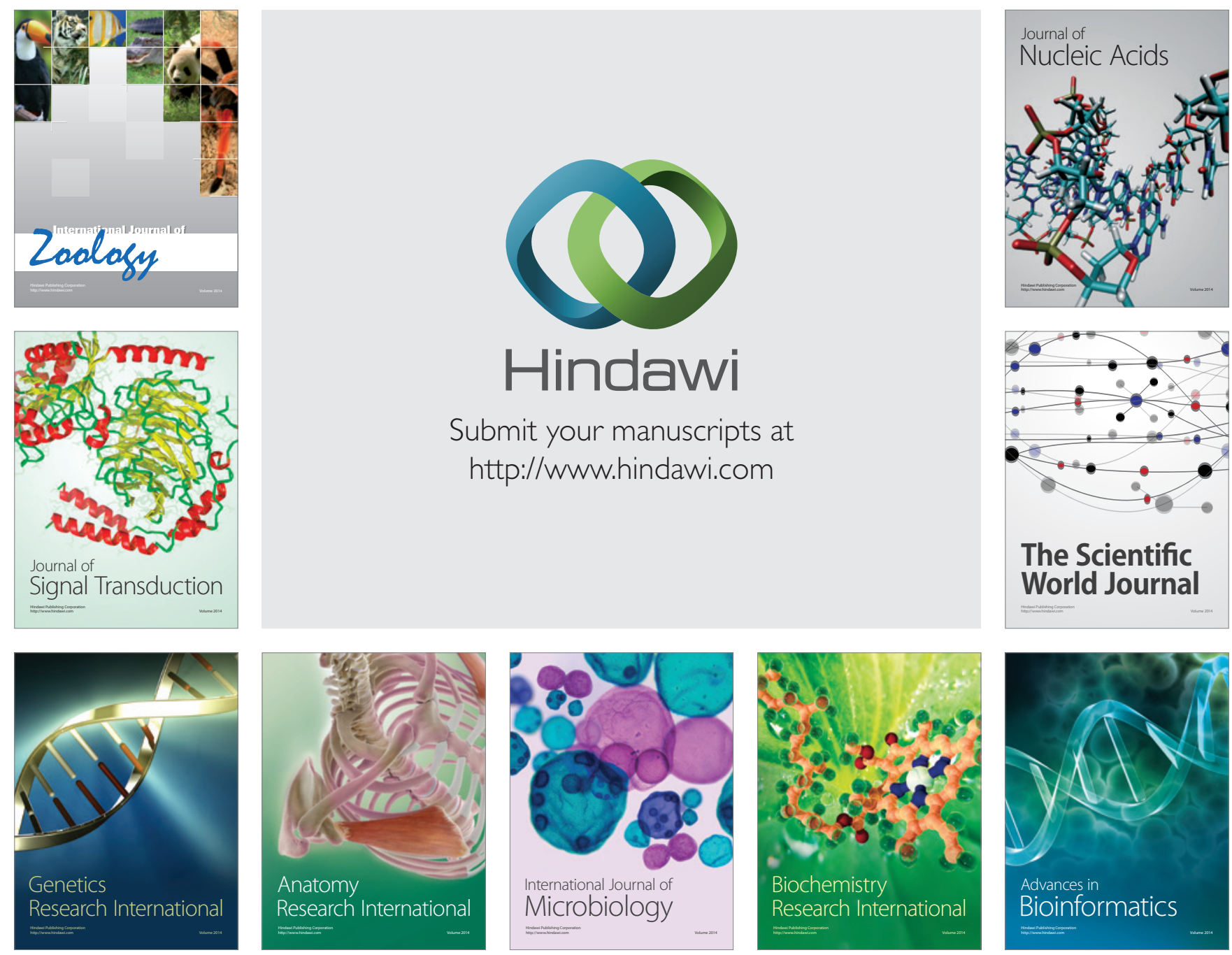

The Scientific World Journal
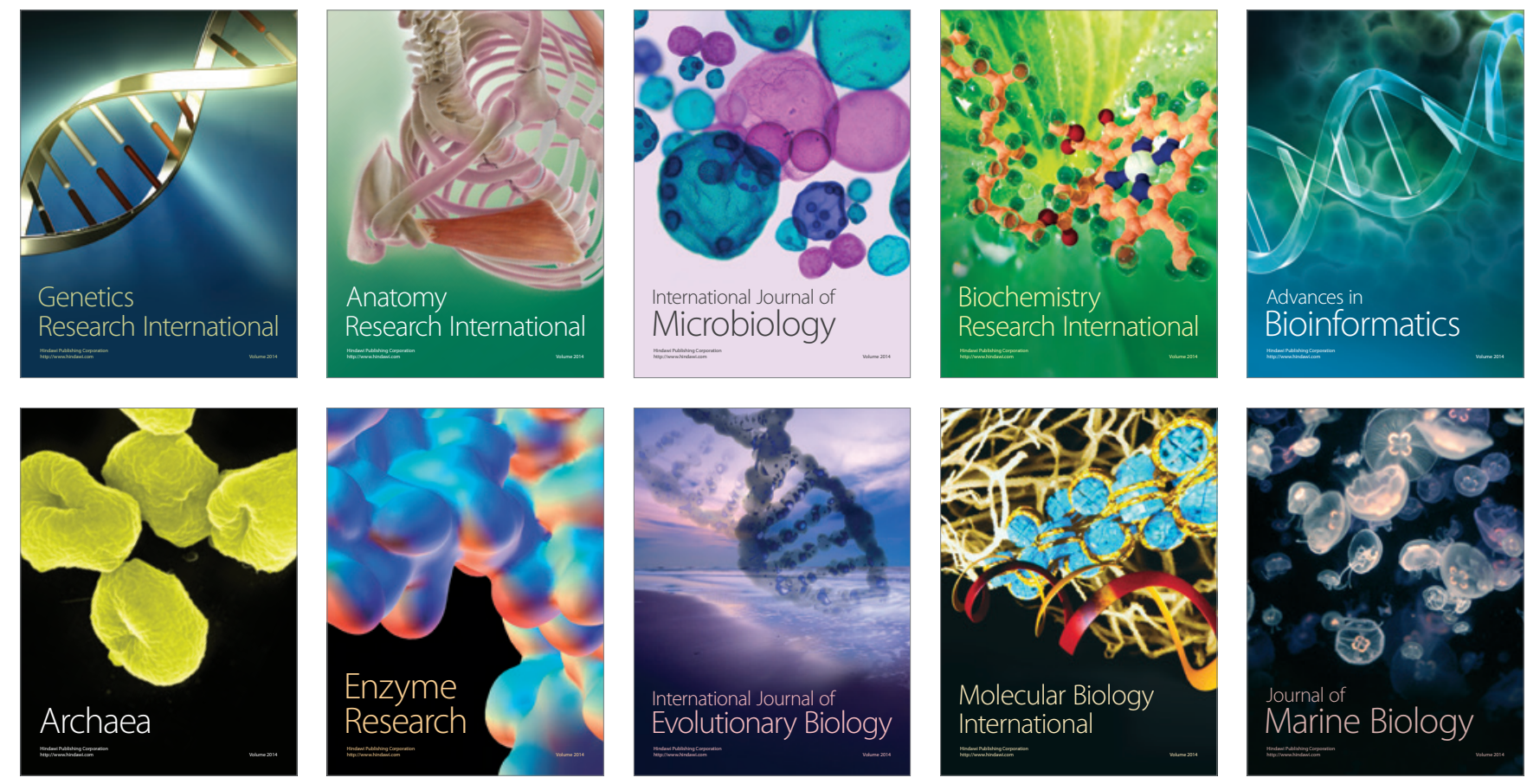